\title{
NEW IMPROVEMENTS IN SHEAROGRAPHY TECHNIQUES
}

\author{
Hernani M. Lopes ${ }^{1}$, Mario A. Vaz ${ }^{2}$, Rui M. Guedes ${ }^{2}$ and J.F. Silva Gomes ${ }^{2}$ \\ ${ }^{2}$ ESTIG - Polytechnique Institute of Braganca, Cp. St Apolonia, Apr. 134 - 5301-857 Braganca \\ ${ }^{2}$ Faculty of Engineering, University of Porto, R Dr Roberto Frias, 4200-465 Porto, Portugal \\ gmavaz@fe.up.pt
}

Shearography is a non-contact full field technique used in the assessment of spatial gradient of displacements through a preset direction, Hung and Taylor [1]. The optical set-up can be based on a Michelson interferometer scheme, for static or quasi-static measurements, and on the MachZehnder interferometer if dynamic measurements have to be performed, Santos et al [2]. In both set-ups a speckle pattern, obtained from a rough surface, is laterally shifted to create the interference and the light used for the interferometer arms have a common path between the object and the set-up. So, this interferometric technique can be used with low coherence lasers and is almost insensitive to rigid body motion. The fringe patterns obtained with shearography result from the interference between two speckled wave fronts and have lower contrast that the ones obtained with specular reference interferometers.

Compact set-ups can be designed which can be used to perform measurements in industrial environments. Image processing techniques can be associated to shearography to improve data analysis and increase the measurement resolution. Phase calculation algorithms, based on temporal or spatial phase shift, are already available and the same stands for phase filtering and unwrapping. New image processing routines were developed to deal with shearography noisy patterns and extract the important information and this technique became an important tool in Non-destructive inspection (NDI) of structures.

The shearography is shown to be a very effective way of measuring the first spatial derivate of the displacements and avoids error propagation through the used of numerical methods. Recent developments in image analysis tools for processing noisy images allowed the assessment of the displacement by integration or the following derivatives by image differentiation, as can be seen in Fig. 1.

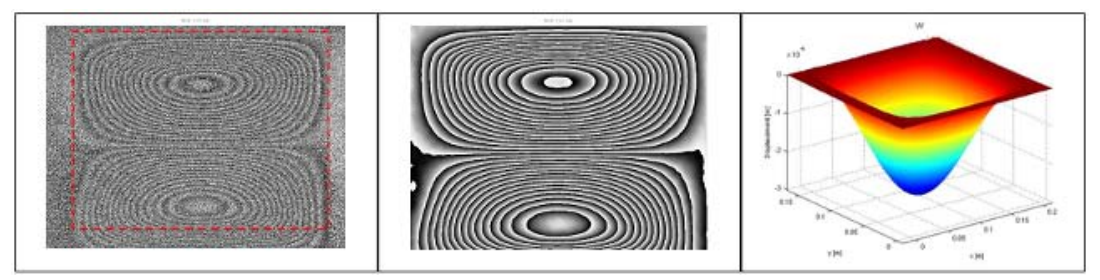

FIGURE 1. Phase-map obtained with shearography, filtered phase and the displacement field

In this work two set-ups for continuous and pulsed illumination measurements with shearography were constructed and tested. All the necessary software for image analysis was implemented and used with experimental data. Several filtering and smoothing algorithms were also tested to obtain data differentiation with good signal to noise ratios, Lopes et al [3][4]. Using an extra mirror in the original Michelsson set-up was possible to obtain up to the third derivative. 
The results obtained with optical and numerical differentiation were compared with the analytical solution as is shown in Fig. 2. The experimental data was obtained by measuring the out of plane displacement gradient of a rectangular composite plate clamped in its entire border and submitted to a uniform pressure.

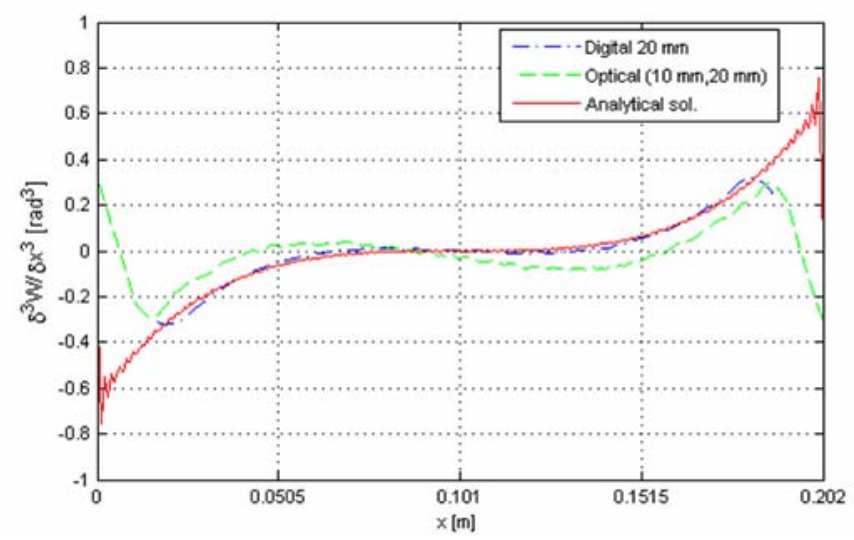

FIGURE 2. Comparison between the analytical solution and the numerical and optical differentiation, for the third displacement derivative.

\section{References}

1. Hung, Y., Taylor, C., A tool for measurement of derivatives of surface displacement. Proc Photo-opt Instrum Eng (SPIE), vol. 41, 169 - 175, 1973

2. Santos, F., Vaz, M.A.P., Monteiro, J., "A new set-up for pulsed digital shearography applied to defect detection in composite structures", Optics and Lasers in Engineering, vol. 42, 131140,2004

3. Lopes, H.M., Guedes, R.M., Vaz, M.A.P., An improved mixed numerical-experimental method for stress field calculation, Optics \& Lasers Technology, vol. 39, 1066-1073, 2007.

4. Lopes, H.M., Guedes, R.M., Vaz, M.A.P., Techniques for numerical differentiation of experimental noisy data, Proc. Of $5^{\text {th }}$ Intern. Conf. on Mechanics and Materials in Design, Ed. J F Silva Gomes \& S A Meguid, Edicoes INEGI, 27 - 28, Porto, 2006 